\title{
Review: Hydrocodes for structural response to underwater explosions
}

\author{
Hans U. Mair \\ Institute for Defense Analyses, Operational \\ Evaluation Division, 1801 North Beauregard Street, \\ Alexandria, VA 22311-1772, USA \\ E-mail:mairh@asme.org \\ Received 7 July 1997 \\ Revised 16 April 1999
}

The applicability of the various hydrocode methodologies (Lagrangian, Eulerian, Coupled Eulerian-Lagrangian, and Arbitrary Lagrangian-Eulerian) for structural response to underwater explosions is reviewed. Only codes employing "structural elements" are realistically applicable to the analysis of thin-walled structural response to underwater explosions.

\section{Overview of hydrocodes}

Computational continuum mechanics is a superset of the largely independent fields of computational fluid dynamics (CFD) and computational solid mechanics (CSM, of which computational structural dynamics, or CSD, is a subset). "Hydrocodes" are computational continuum mechanics tools that simulate the response of both solid and fluid material under such highly dynamic conditions (e.g., detonation and impact) that shock wave propagation is a dominant feature. Hicks and Walsh [38], Anderson [4], Benson [9], and McGlaun and Yarrington [71] review the numerical features of hydrocodes; Johnson and Anderson [48] review hydrocode applicability to hypervelocity impact problems.

Hydrocodes have provided more than simply "hydrodynamic" simulations of material behavior for a long time, but in keeping with the common terminology, the misnomer "hydrocodes" is employed. An alternate term, though less widespread, is "wavecodes," due to the wave-capturing nature of these codes. The term "hydrocodes" is reserved by some for the Eulerian versions of the codes. Hydrocodes make fewer ap- proximations than either of the more special-purpose CFD or CSM methods. They numerically solve the more fundamental time-dependent equations of continuum mechanics (compared to, for example, the Navier-Stokes equations of fluid dynamics), thereby fulfilling requirements for which neither traditional CFD nor CSM codes are fully suitable. The everexpanding scope of CFD, CSM, and hydrocode capabilities has, however, created a semantic difficulty: it is impossible to delineate strictly between hydrocodes and either CFD or CSM codes. Hydrocodes are therefore here defined as tools for the simulation of multimaterial, compressible, transient continuum mechanics (i.e., mechanical wave propagation through multiple fluids and solids). Some hydrocodes have even implemented the capability to practically model the dynamics of thin-walled structures, allowing an even wider applicability. This incorporation of traditional structural analysis capabilities into hydrocodes is crucial to simulating the response of engineering structures to underwater explosions.

The development of hydrocode capabilities was originally driven by military requirements related to explosives and high-velocity impact. However, this class of tools has more recently found commercial applicability (and corresponding development incentive) in such diverse fields as automobile and shipping container crashworthiness (Du Bois [22]; Frank and Gruber [28]; Belytschko et. al. [8]; Logan, Tokarz and Whirley [60]), automobile airbag deployment (Du Bois [22]; Choi [15]), automobile and train occupant reaction to crashes (Du Bois [22]), bird impact on aircraft (Du Bois [22]; Luttwak, Florie, and Venis [62]), and sheet metal forming (Galbraith, Finn and MacEwen [30]; Galbraith and Finn [31]; Couch et al. [19]).

The Lagrangian, Eulerian, Coupled Eulerian-Lagrangian (CEL), and Arbitrary Lagrangian-Eulerian (ALE) hydrocode methods are described in the sections that follow. A simple illustration is included for each method, depicting a structure-medium interaction (SMI) whose geometry is illustrated in Fig. 1. It rep- 

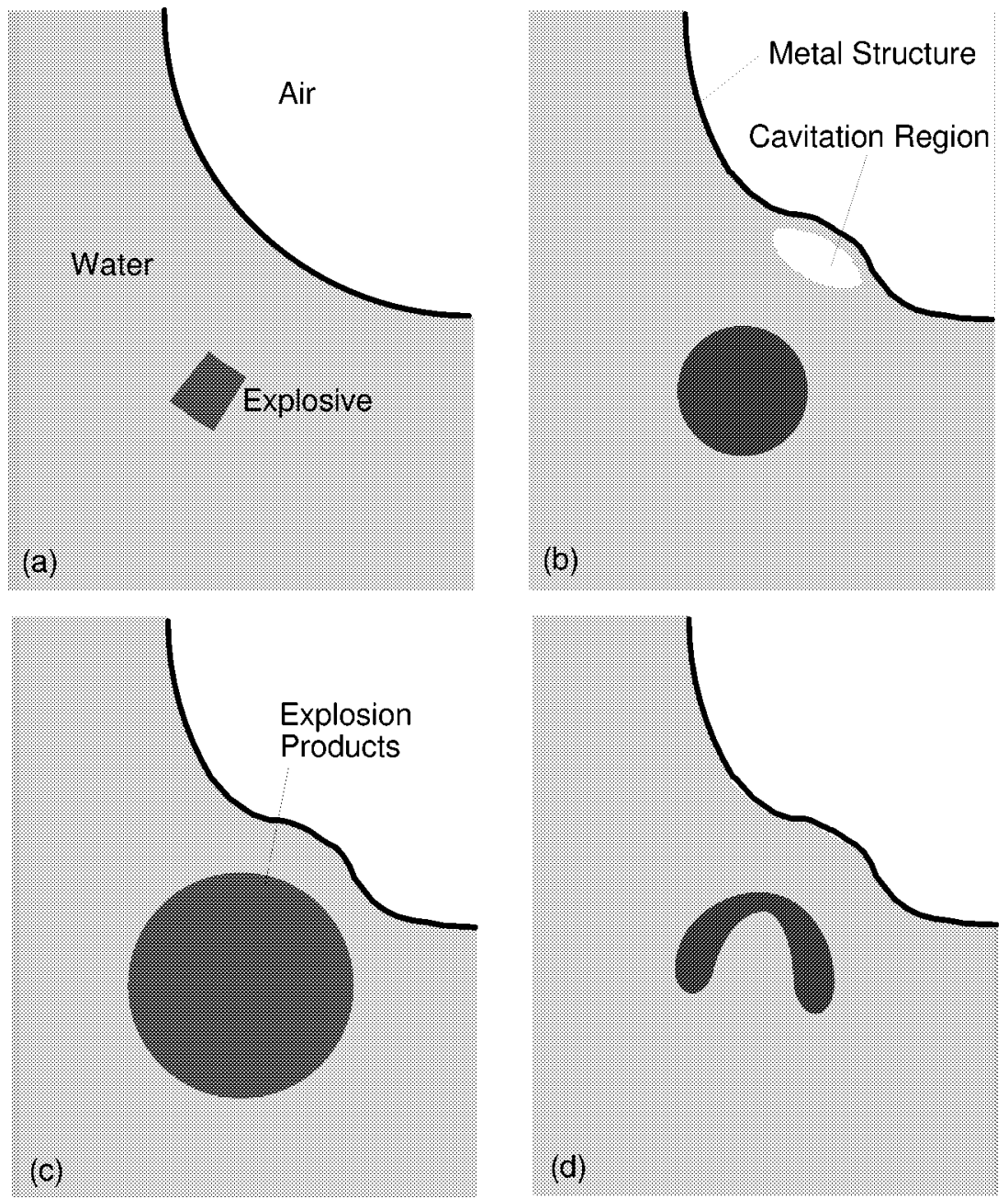

Fig. 1. UNDEX-SMI analysis example.

resents a two-dimensional analog of the type of problem that challenges most aspects of underwater explosion structure-medium interaction (UNDEX-SMI): an underwater explosion that causes large structural distortion and displacement and in which bubble collapse and cavitation effects are significant. The early expansion of the explosion products sends a strong shock wave into the surrounding water. The shock quickly deforms the structure, resulting in a cavitation region in the water close to the structure. The cavitation region eventually closes as the bubble continues to expand, and a water jet is shown to form as the bubble collapses. None of the examples are actual computations; they only illustrate the capabilities and limitations of the methods. All hydrocode methods are theoretically capable of modeling structure-medium interaction since no major coupling approximations are made. The methods are, therefore, judged on practicality instead of capability. The individual code developers should be consulted for up-to-date code capabilities and projected improvements.

\section{Lagrangian hydrocodes}

The computational mesh of a Lagrangian model remains fixed on the material, as illustrated in Fig. 2. Since the mass within each element remains fixed, no mass flux at interelement boundaries must be computed; thus the computation is relatively straightforward and fast. Material distortions correspond to Lagrangian mesh distortions, leading to reductions in time steps and/or breakdown in problem advancement. Mesh rezoning tends to extend the application of La- 

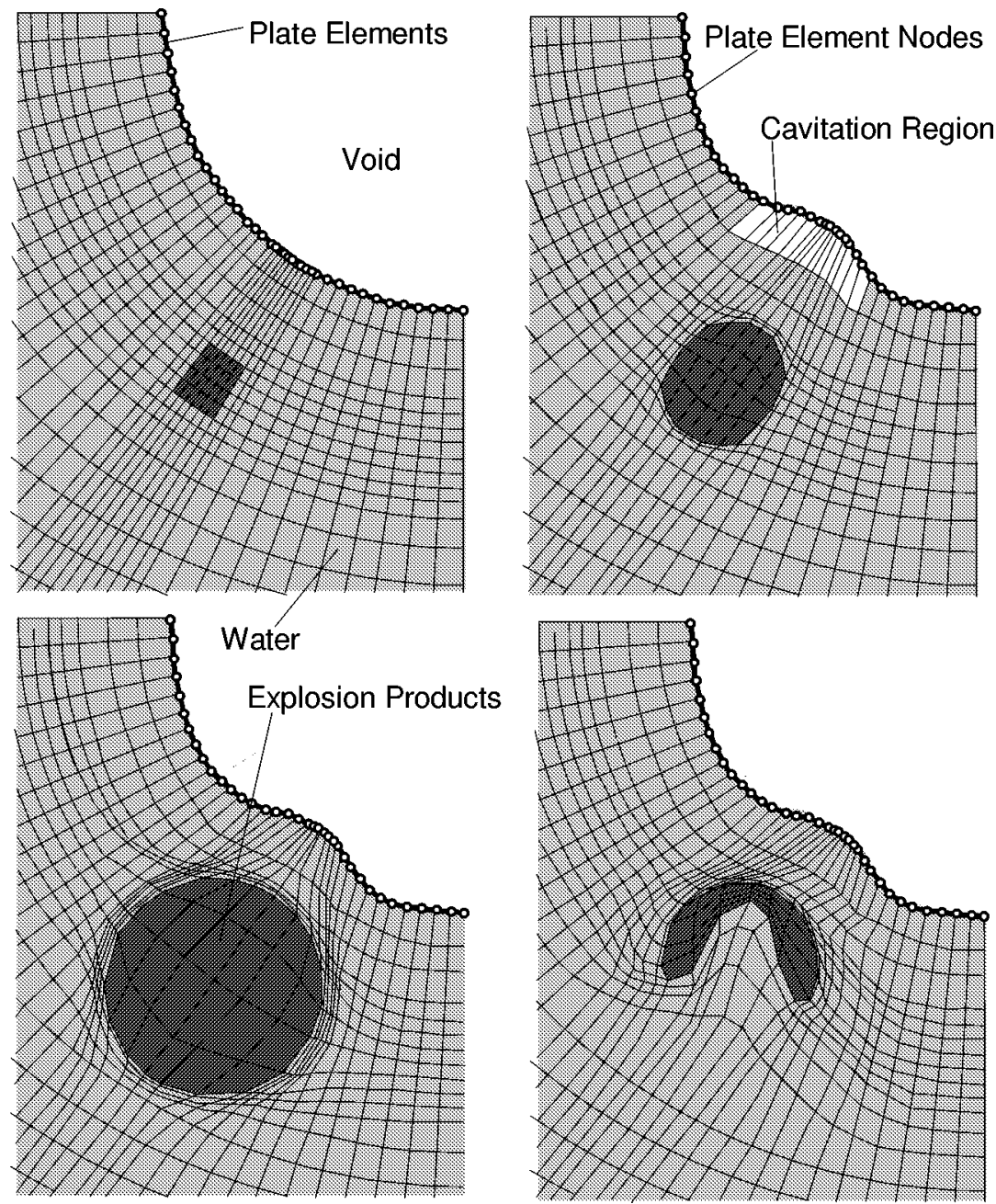

Fig. 2. Lagrangian hydrocode UNDEX-SMI example.

grangian codes to large distortion problems, but introduces complexities and corresponding solution inaccuracies. The calculation is also no longer strictly Lagrangian if the mesh is rezoned. The distorted (elongated) elements defining the water at the edge of the bubble in Fig. 2 are apparent. These distorted elements would likely cause the accuracy and time step to drop to unacceptable values, in effect stopping the calculation. The unfavorable mesh evolution is due, in part, to the quadrilateral elements (six-sided "hexahedral" elements in 3D); triangular elements (four sided "tetrahedral" elements in 3D) tend to be more forgiving of large distortions, though numerical problems must be carefully controlled. This point is illustrated by the fact that shaped charge liner collapse is commonly and effectively modeled using codes that employ triangular/tetrahedral elements. Even if more "ro- bust" elements were employed, the Lagrangian method would still ultimately break down as the bubble collapses upon itself, since the mesh that defines the explosion products cannot simply disappear. The general limitation of most Lagrangian hydrocodes to relatively low-distortion computations limits their applicability to shock-structure interaction analysis.

Predecessors of modern Lagrangian hydrocodes were HEMP (Wilkins [100], TOODY (Swegle [88]), 2DL (Johnson, Mader and Shaw [49]) and HONDO (Key [54]). EPIC (Johnson et al. [47]), various versions of DYNA (Hallquist et al. [34-36]; Whirley and Hallquist [99]) and PRONTO (Taylor and Flanagan [92,93]; Attaway [6]; Bergmann [11]) are the most prominent Lagrangian hydrocodes, but the Lagrangian modules of the Coupled Eulerian-Lagrangian (CEL) codes (described in a subsequent section) are 


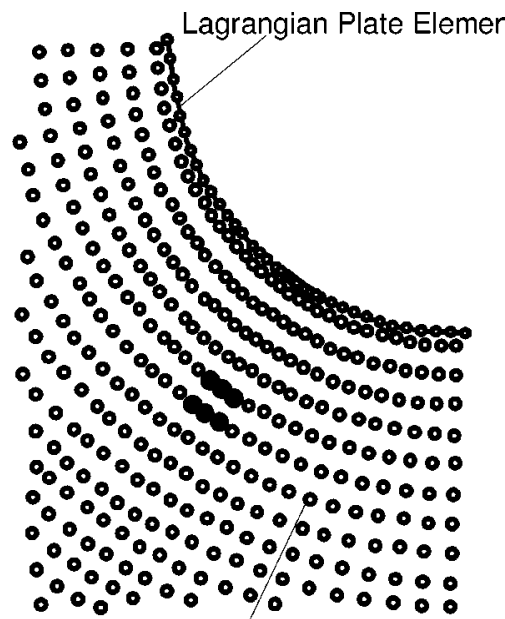

Water Particles

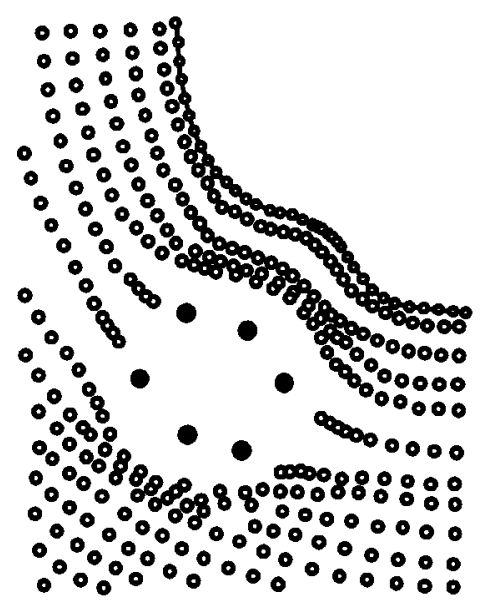

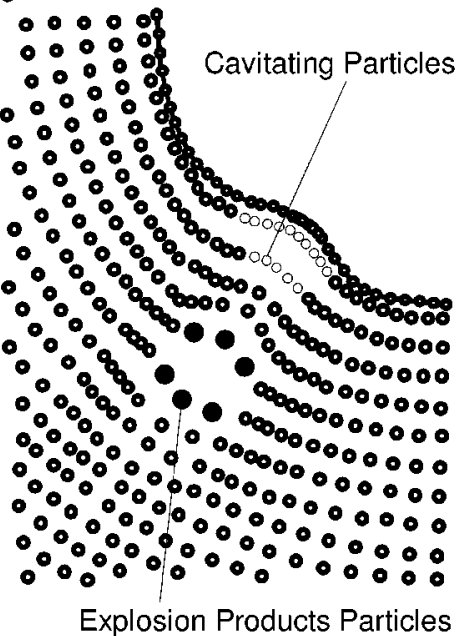

Explosion Products Particles

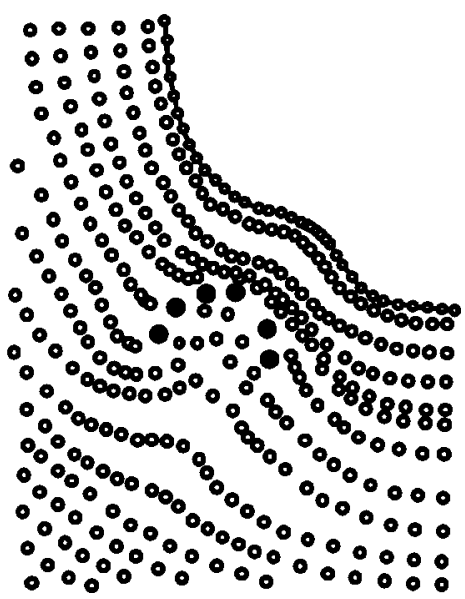

Fig. 3. SPH-Lagrangian hydrocode UNDEX-SMI example.

also available. Any Arbitrary Lagrangian-Eulerian (ALE) code (described below) can also be run in purely Lagrangian mode. Traditional (implicit) Lagrangian solid/structural mechanics codes like NASTRAN (MSC [73]), NIKE (Englemann and Hallquist [24]; Maker, Ferencz, and Hallquist [64]), and ABAQUS (HKS [39]) are not suitable, or not efficient, for highly dynamic analyses in which shock waves are dominant. The EPIC code is based primarily on triangular elements in 2D and tetrahedral elements in 3D, and is therefore less subject to mesh distortion problems. Several attempts have been made at coupling the strictly Lagrangian hydrodynamic and structural capabilities of DYNA3D for UNDEX-SMI analysis (Mair et al. [63]; Kwon, Fox, and Shin [56]).

Lagrangian hydrocodes have been successfully coupled and linked to Eulerian hydrocodes such that large distortion fluid dynamics calculations can be made within an Eulerian framework. The Arbitrary Lagrangian-Eulerian (ALE) method, also described in a following section, can be considered a variant (or even superset) of the Lagrangian method.

Other Lagrangian methods that have potential application to UNDEX-SMI analysis but are not as mature as the standard method include the Free Lagrange Method (FLM) (Fritts, Crowley, and Trease [29]; Brackbill and Monaghan [12]), Smoothed Particle Hydrodynamics (SPH) (Benz [10]; Libersky et al. [59]; Petschek and Libersky [77]), and the Total Lagrangian formulation (Sandler [82]). A Free Lagrange Method (FLM) mesh distorts as the material distorts, but the connectivity of the elements changes as the mesh distorts. This concept is illustrated in Fig. 4, which could illustrate the mesh distortions accompanying shear banding. The elements that define the domain redefine their connectivity as the mesh distorts; this is 


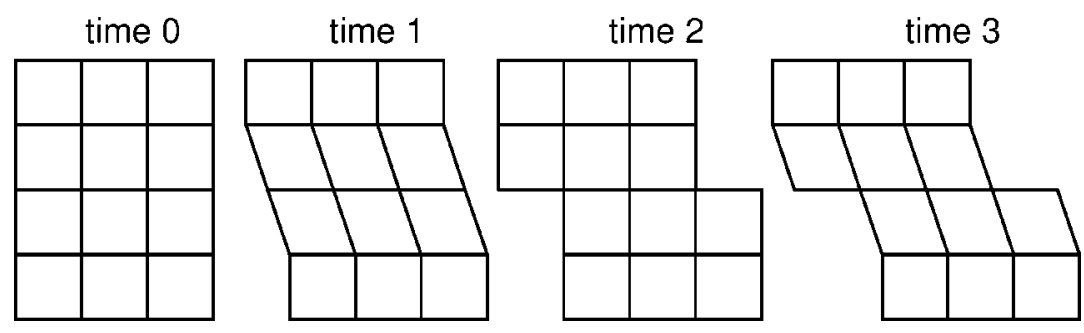

Fig. 4. A free-lagrange mesh motion example.

not possible in a standard Lagrangian code. Research codes that are examples of FLM include CAVEATGT (Dukowitz, Cline, and Addessio [23]) and TRIX (Gittings [32]). In Smoothed Particle Hydrodynamics (SPH), Lagrangian "particles" dynamically interact with no fixed connectivity, as shown in Fig. 3. Lagrangian hydrocodes that have implemented SPH as an analysis option include EPIC (Johnson, Peterman, and Stryk [45]; Johnson [46]), PRONTO (Swegle and Attaway [90]) and AUTODYN-2D (Clegg et. al [17]). In the Total Lagrangian (vice "Updated Lagrangian") formulation, as implemented in the FUSE Lagrangian hydrocode (Sandler [82]), Lagrangian elements with fixed connectivity operate on individually determined time steps, thereby overcoming the traditional limitation that one small element with the shortest length scale determines the global time step. The FLM and SPH methods are particularly attractive for large shear distortion and material separation problems, overcoming the main limitation of traditional Lagrangian hydrocodes. An UNDEX-SMI event would probably be modeled using Lagrangian particles, FreeLagrange elements, or Total Lagrangian elements to trace fluid motions and continuously interact with traditional Lagrangian structural elements (Fig. 3). Since the "mesh" of SPH and FLM codes is not rigidly interconnected, the methods do not break down as an underwater explosion bubble collapses upon itself. UNDEX-SMI simulations have been attempted with the PRONTO SPH-Lagrangian hydrocode (Swegle and Attaway [90]) and with FUSE (Sandler [82]).

The natural applicability of Lagrangian hydrocodes with structural analysis capability to highly dynamic problems spawned the automobile crashworthiness simulation industry. Many explicit codes are currently applied to, and developed explicitly for, automobile crashworthiness; LS-DYNA (LSTC [61]), ABAQUS/Explicit (HKS [40]), and PAM-CRASH (ESI [25]) are examples. Two significant features of crash simulation codes are advanced contact algorithms (for structural members folding upon them- selves) and Adaptive Mesh Refinement (AMR) algorithms, since many plastic hinges form.

\section{Eulerian hydrocodes}

Eulerian hydrocodes advance solutions in time on a mesh fixed in space, as illustrated in Fig. 5, instead of a mesh fixed on the material, as is done in a Lagrangian solution. By advancing the solution on a computational mesh fixed in space and time, Eulerian codes avoid the Lagrangian problem of mesh distortions. Correspondingly, and unlike Lagrangian simulations, time steps can remain roughly constant during simulations, and bubble jetting simulations become feasible. The difference between Eulerian computational fluid dynamics (CFD) codes and Eulerian hydrocodes is primarily the inclusion of material strength (flow of solids) and multimaterial capability in the hydrocodes. Furthermore, Eulerian hydrocodes are strictly transient dynamics solvers; they are not designed to solve steadystate fluid flow problems.

The usual solution sequence for every time step within Eulerian hydrocodes consists of a Lagrangian computation, followed by a remap (or "advection") phase, which restores the slightly distorted mesh to its original state. This process is unlike the traditional Eulerian CFD approach, in which materials are transported through the fixed mesh in one step by solving the equations of motion in their Eulerian form. The transport of material interfaces and materials with strength requires sophisticated algorithms that can strongly influence the solution.

Cells (or elements) containing more than one material are common in Eulerian hydrocode computations; the presence of multiple fluid and/or solid materials within a cell is the main reason that these codes are computationally expensive, and sets them apart from more traditional CFD codes. This situation calls for numerical algorithms that prevent artificial material diffusion (the mixing of materials across a mate- 
Mixed Water/Steel/Void Cells

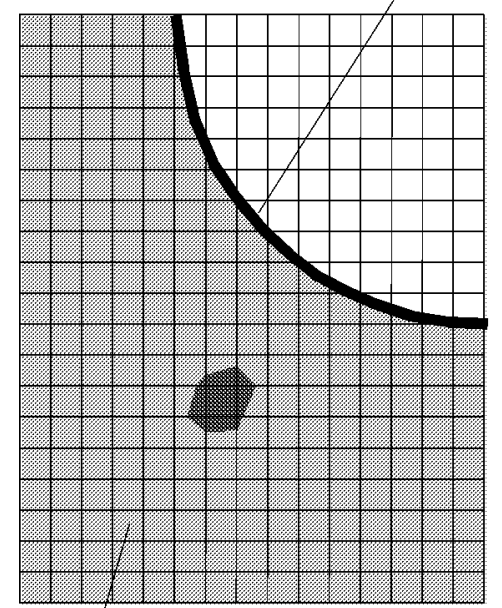

Mixed Water/Explosion Products Cells
Void Eulerian Cells

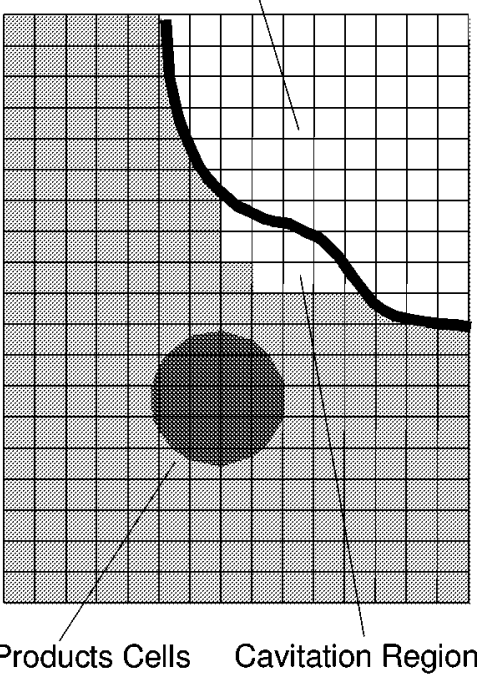

Cavitation Region

\section{Water-Filled Cells}
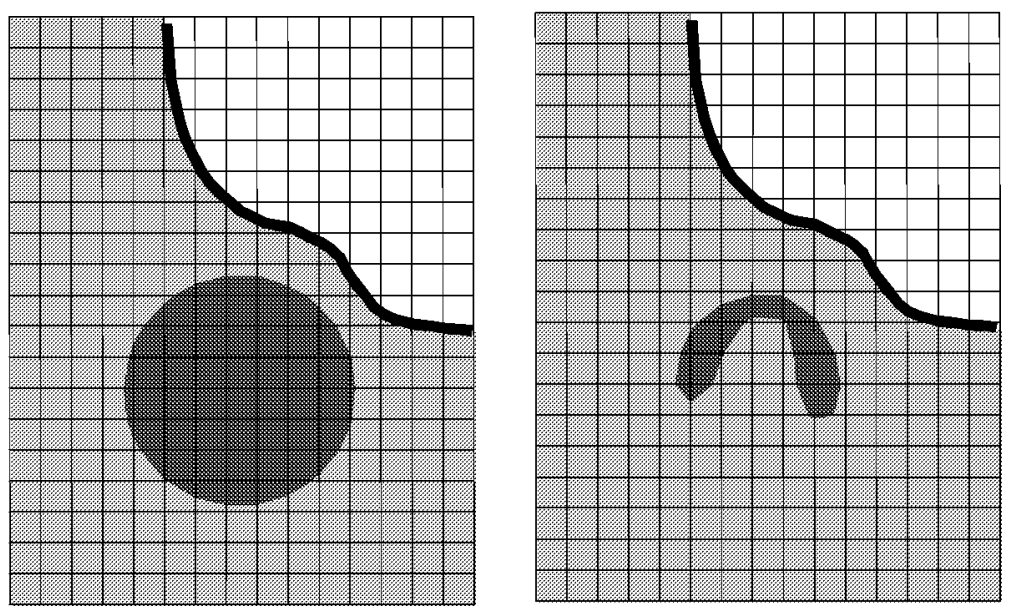

Fig. 5. Eulerian hydrocode UNDEX-SMI example.

rial interface) within these mixed cells. The convergence to a common state parameter (e.g., pressure) within a multimaterial cell can also result in considerable expense, particularly if the higher order accuracy of the Lagrangian phase is to be retained. The additional computational effort expended in the remap phase is largely responsible for the discrepancy in comparative Eulerian versus Lagrangian computational expense.

Since solid materials are defined within Eulerian cells whose properties must be uniform, several modeling limitations present themselves. Several cells must define the thickness of any plate structure to capture its bending stresses; this necessitates prohibitively fine zoning. Furthermore, structural properties of a solid will be "smeared" with the fluid properties of any adjacent water within a mixed cell. Combined, these facts result in Eulerian UNDEX-SMI computations being highly impractical.

The most prominent Eulerian hydrocode is CTH (McGlaun et al. [70]; McGlaun [67,68]; McGlaun, Thompson, and Elrick [69]; Bell et al. [7]; Silling [8486]; Kerley [52,53]; Taylor [94-96]; Crawford [21]; Farnsworth [26]) and a massively parallel implementation PCTH. Other Eulerian hydrocodes include MESA (Holian et al. [41]; Mandell et al. [65]) and its massively parallel implementation PAGOSA (Kothe et al. [55]), TECOLOTE (Holian et al. [42]), GRIM (Church et al. [16]), and RAGE (SAGE) (Allen, Lumsden, and Zerkle [2]; LANL [57]). The Eulerian modules of the 
Coupled Eulerian-Lagrangian (CEL) codes, described below, are also commonly used. Also, the more general type of Arbitrary Lagrangian-Eulerian (ALE) codes (see below) can be run in purely Eulerian mode. Since computational structural dynamics analysis is highly impractical in an Eulerian frame, no examples of Eulerian UNDEX-SMI analyses exist.

Eulerian hydrocodes are related to the more traditional Eulerian computational fluid dynamics (CFD) codes, some of which have even demonstrated their ability to model underwater explosion bubbles (Kan and Stuhmiller [50]; Rogers and Szymczak [81]; Szymczak [91]). Eulerian hydrocodes have been successfully coupled to Lagrangian hydrocodes such that detailed structural calculations can be made within a Lagrangian framework (see "Coupled Eulerian-
Lagrangian (CEL) Hydrocodes", below). The Arbitrary Lagrangian-Eulerian (ALE) method, described in a following section, can be considered a variant of the Eulerian method; in effect, the Eulerian method is a subset of the ALE method.

\section{Coupled Eulerian-Lagrangian (CEL) hydrocodes}

Coupled Eulerian-Lagrangian (CEL) hydrocodes employ both Eulerian and Lagrangian methods in their most advantageous modes in separate (or overlapping) regions of the domain. As illustrated in Fig. 6, the Lagrangian shell elements continuously interact with the Eulerian fluid domain. A recommended practice con-

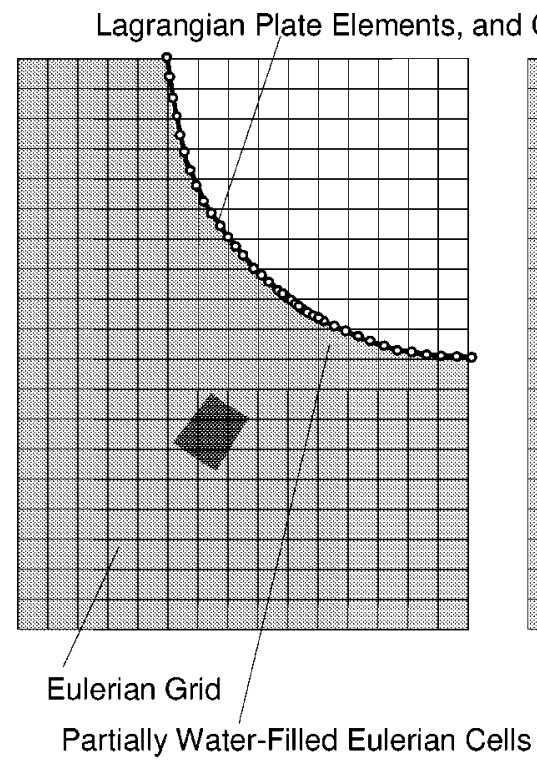

d Coincident Interface "Elements"

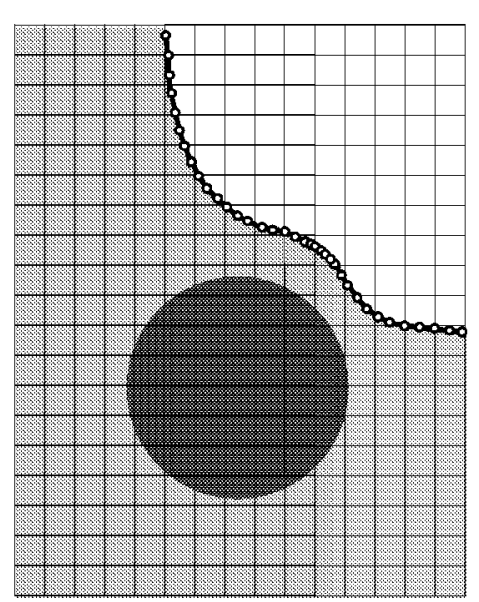

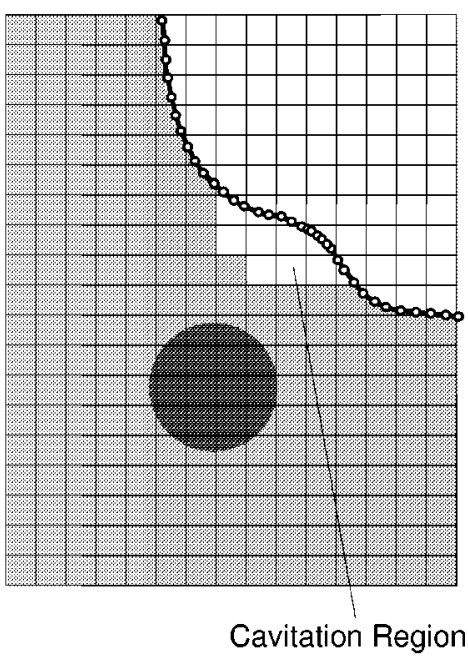

Void Eulerian Cells

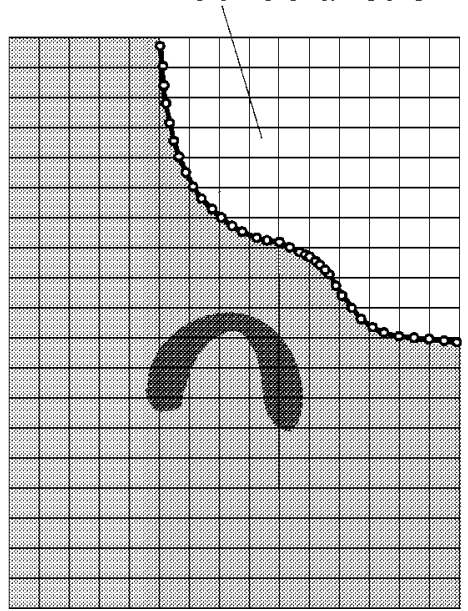

Fig. 6. Coupled Eulerian-Lagrangian hydrocode UNDEX-SMI example. 
cerning the application of the CEL method is to discretize solids (materials in which the material strength plays a dominant role) in a Lagrangian frame, and materials exhibiting primarily fluid behavior (little or no strength) in an Eulerian frame. The Eulerian and Lagrangian regions continuously interact with each other, allowing true coupling of a fluid and a structure. A typical CEL code actually comprises three modules: Eulerian, Lagrangian and Coupling. The Coupling module handles the two-way flow of information between the Eulerian and Lagrangian modules in one of two ways.

In the first coupling method, "interface elements" are employed that coincide with the exterior surfaces of the Lagrangian model, forming a surface in a three dimensional model (a line in a two-dimensional model). The interface elements determine the volume of the Eulerian cells partially covered by the Lagrangian mesh. The presence of an arbitrarily shaped Lagrangian model usually creates some very small volumes in partially covered Eulerian cells; these are "blended" with neighboring cells to avoid the severe time step restrictions on small cells. This coupling method is used in DYSMAS/ELC (IABG [44]), PISCES (Pohl et al. [79]; Hancock [37]; Groenenboom [33]), and DYTRAN (Keene and Prior [51]).

The second coupling method avoids the difficulty of calculating partially filled cells by including the Lagrangian material within the Eulerian calculation. Since the dynamics of thin materials cannot be calculated practically within an Eulerian mesh, this coupling method has not been applied to UNDEX-SMI analysis. This method is applicable to high-velocity impact analyses; it is employed in HULL (Matuska and Osborne [66]) and the coupling of CTH with EPIC through ZAPOTEC (Yarrington [101]; Yarrington and Prentice [102]; Prentice [80]).

All CEL codes can compute in a strictly Lagrangian or Eulerian mode. A feature of some CEL codes is the capability to rezone a Lagrangian mesh into an Eulerian mesh. An example of this is to begin the computation of a shaped-charge liner in a Lagrangian frame, and to convert the liner into an Eulerian computation after material distortions have made the continuation of Lagrangian computations impractical.

An example of an UNDEX-SMI event that a CEL code would find difficult or impossible to model properly would be where thin structural members extend out into the fluid, as illustrated in Fig. 7. A CEL code must define the interface between the Lagrangian region (structure) and Eulerian region (fluid), but a La-

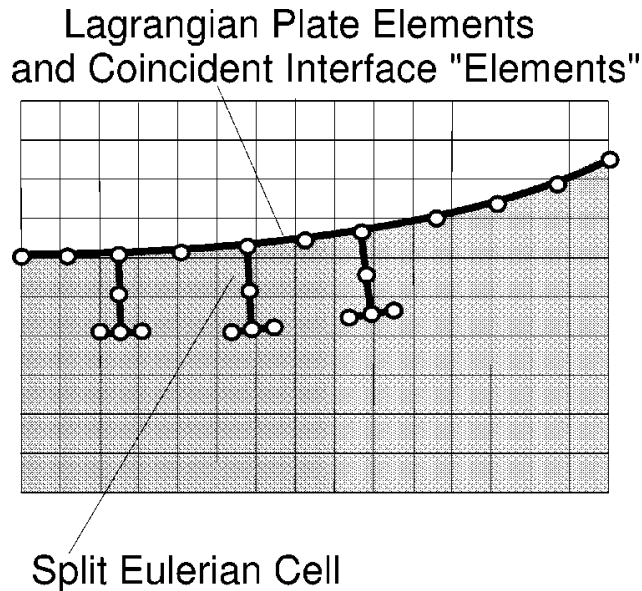

Fig. 7. A difficult coupling problem for CEL codes.

grangian structural element (no spatial thickness) can easily "see" a single Eulerian fluid cell on both sides. Thus two separated fluid regions would be described within a single Eulerian cell - an unphysical situation. The employment of Lagrangian continuum elements for the structure would tend to overcome this problem, but then the disadvantages of using continuum elements where structural elements are more appropriate come into play.

The original Coupled Eulerian-Lagrangian code was CEL (Noh [75]). MSC/DYTRAN (formerly PISCES-3DELK) (Pohl et al. [79]; Keene and Prior [51]), MSC/PISCES (formerly PISCES-2DELK) (Hancock [37]; Groenenboom [33]), HULL (Matuska and Osborne [66]), DYSMAS/ELC (IABG [44]), AUTODYN-2D (Century Dynamics [13]) and the coupling of CTH with EPIC through ZAPOTEC (Yarrington [101]; Yarrington and Prentice [102]; Prentice [80]) are the primary CEL hydrocodes in use. All except the HULL and CTH/EPIC codes have some practical thin-plate structural analysis capability in 2D or 3D. MSC/DYTRAN and DYSMAS/ELC have 3D coupled capability using "interface elements", with substantial structural analysis capability. They are therefore the CEL codes currently capable of practical, three-dimensional UNDEX-SMI simulation. The capability for modeling the interaction between underwater explosions and 3D naval structures has been demonstrated with the CEL method using DYSMAS/ELC (Pfrang, Luetje, and Freercks [78]; Schittke et al. [83]; Andelfinger [3]; McKeown, Grande, and Mentges [72]) and MSC/DYTRAN (Lenselink and DeVries [58]; Chisum and Shin [14]); these interactions focus on shock-structure interaction. The capability to 
model both shock-structure and bubble-structure interaction in $2 \mathrm{D}$ has been demonstrated by PISCES2DELK (Huang and Kiddy [43]) and DYSMAS/ELC (Wardlaw, Fiessler, and Han [98]; Fiessler, Han, and Wardlaw [27]).

\section{Arbitrary Lagrangian-Eulerian (ALE) hydrocodes}

Arbitrary Lagrangian-Eulerian (ALE) hydrocodes share aspects with both Lagrangian and Eulerian hydrocodes; Lagrangian motion is computed every time step, followed by a remap phase in which the spatial mesh is either not rezoned (Lagrangian), rezoned to its original shape (Eulerian) or rezoned to some more "advantageous" shape (between Lagrangian and Eulerian). In this way the spatial description of the mesh is neither restricted to following material motions (Lagrangian) nor remaining fixed in space (Eulerian). ALE mesh motions are based primarily on the preservation of a uniform mesh, not the capture of physical phenomena.

The ALE method provides a way of coupling fluid dynamics to structural dynamics without interfacing two separate coordinate systems as is done in the Coupled Eulerian-Lagrangian method. The efficiency to be gained by straightforward coupling (the avoidance of a separate coordinate coupling module) is probably significant. In cases where structural elements can be incorporated directly within the ALE framework, the coupling is trivial. In cases where the ALE numerical method cannot incorporate structural elements, the coupling can be effected through a continuous transfer of boundary conditions without the coordinate system interactions required in the CEL method.

Two levels of ALE technology exist. One allows ALE behavior only within a material (forcing material boundaries to remain Lagrangian); an example of this "single material" or "Simple" ALE (SALE or SMALE) type is illustrated in Fig. 8. The material boundary between the explosion products and the water remains Lagrangian, and the mesh that defines the water remains uniform (unlike the fully Lagrangian water in Fig. 2). The distinction between the mesh motions of this example and those of the purely Lagrangian example lies primarily in the elements that define the water region adjacent to the expanding bubble, as illustrated in Fig. 9. In the purely Lagrangian case, those elements become exceedingly thin as the bubble expands. In the single material ALE case, those water elements are continuously rezoned such that a much more uniform mesh evolves; this has the advantage that the time step does not drastically drop as it does in the purely Lagrangian case. A disadvantage of rezoning includes the computational expense involved in rezoning from which purely Lagrangian computations are spared. This method, like the fully Lagrangian case, ultimately breaks down as the bubble collapses upon itself, since the mesh that defines the explosion products cannot simply "get out of the way".

The second level of ALE technology allows multimaterial elements to form and is therefore more generally applicable; an example of this "multimaterial ALE" is illustrated in Fig. 10. In this example, most of the mesh remains fixed in space (Eulerian), but the region adjacent to the deforming structure deforms with the structure (Lagrangian). The elements that represent the region between the structure (Lagrangian) and the stationary mesh (Eulerian) are therefore neither Lagrangian nor Eulerian; they form a bridge between the two regions. Since the mesh in the bubble region is primarily Eulerian, this method does not break down as the bubble collapses upon itself.

Structure-medium coupling is generally more efficient in ALE than in CEL codes, since the interface between the structure and its surrounding medium is a Lagrangian boundary for both regions. In fact, an ALE code with structural analysis capability would potentially be able to solve the problem shown in Fig. 7, which a CEL code would find difficult or impossible to model properly. However, the coupling of fluid and structural regions through matching of element nodes creates another problem. As illustrated in Fig. 11, elements that must remain Lagrangian (e.g., plates) cannot be allowed to collapse upon each other if material is entrained between them. This is the same limitation that prevents the Lagrangian and Single-Material ALE (SALE) methods from being applied to bubble jetting problems: the mesh that defines the entrained fluid cannot simply "get out of the way" of a Lagrangian interface, even if the fluid itself could.

The first attempts at creating ALE hydrocodes can be seen in the Lagrangian hydrocodes that implemented "automatic rezoning" options within material boundaries (single material ALE, or SALE). Such codes include ARTOO (based on TOODY) (Swegle [89]) and DYNA2D (Hallquist [34]). VEC/ DYNA3D (Hallquist and Stillman [36]), LS-DYNA (LSTC [61]), and AUTODYN-3D (Century Dynamics [13]) include SALE capability.

Multimaterial ALE hydrocodes include CALE (Tipton [97]), CAVEAT (Addessio et al. [1]), ALE3D (An- 

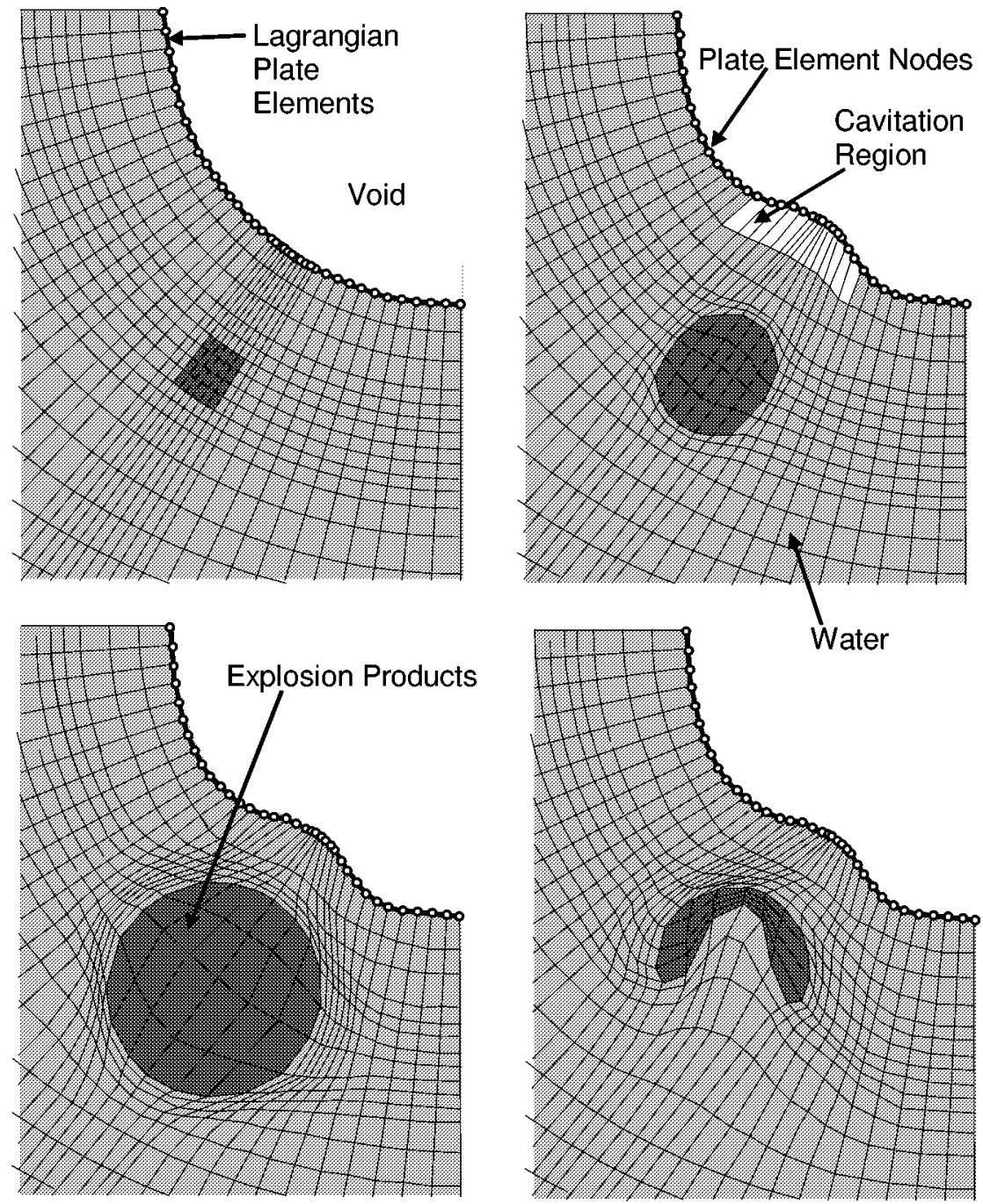

Fig. 8. Single material ALE hydrocode UNDEX-SMI example.

derson et al. [5]; Neely et al. [74]; Couch et al. [20]), ALEGRA (formerly RHALE) (Peery et al. [76]), MSC/DYTRAN (Keene and Prior [51]) and LS-DYNA (LSTC [61]). ALE3D, ALEGRA, DYTRAN and LSDYNA include Lagrangian structural (plate) elements and are therefore applicable to the computational analysis of UNDEX-SMI. Multimaterial ALE UNDEXSMI analyses have been attempted with ALE3D (Couch and Faux [18]) and DYTRAN (Lenselink and DeVries [58]).

The ALE method can be considered a superset of both the Eulerian and Lagrangian methods, since both types of mesh motions are incorporated within an ALE scheme. The ALE method cannot, however, be considered a superset of the Coupled Eulerian-Lagrangian (CEL) method, since the ALE method makes no provi- sion for allowing an Eulerian region to interact directly with a Lagrangian interface. ALE hydrocodes are more general than ALE CFD codes, in that ALE hydrocodes allow simulations of materials with strength.

\section{Summary}

Many simulations of structural response must include the dynamics of the surrounding medium. Such are the intended capabilities of the "hydrocodes", computational mechanics codes that make minimal assumptions about the physical phenomena exhibited by the many classes of problems they are applied to. Detonation physics, shock wave propagation, bubble dynamics, and large-strain structural plasticity and fail- 


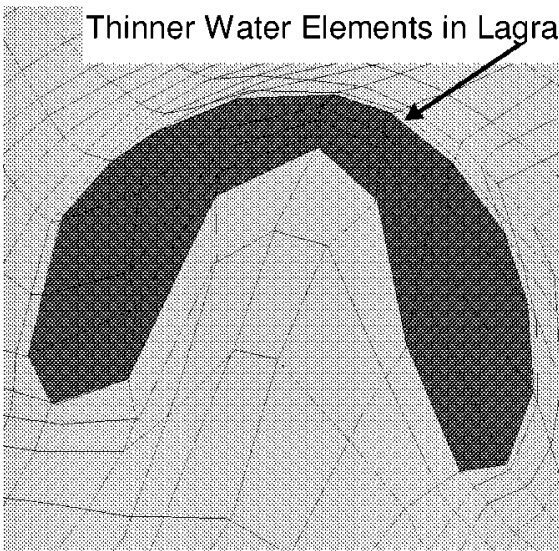

Lagrangian

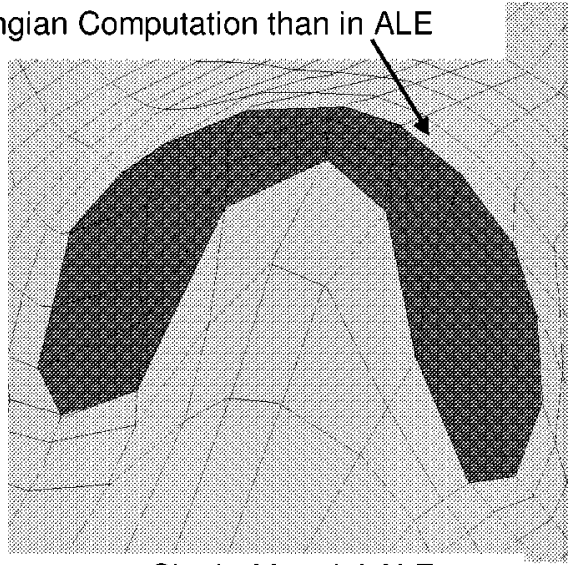

Single-Material ALE

Fig. 9. Comparison of Lagrangian and single-material ALE.
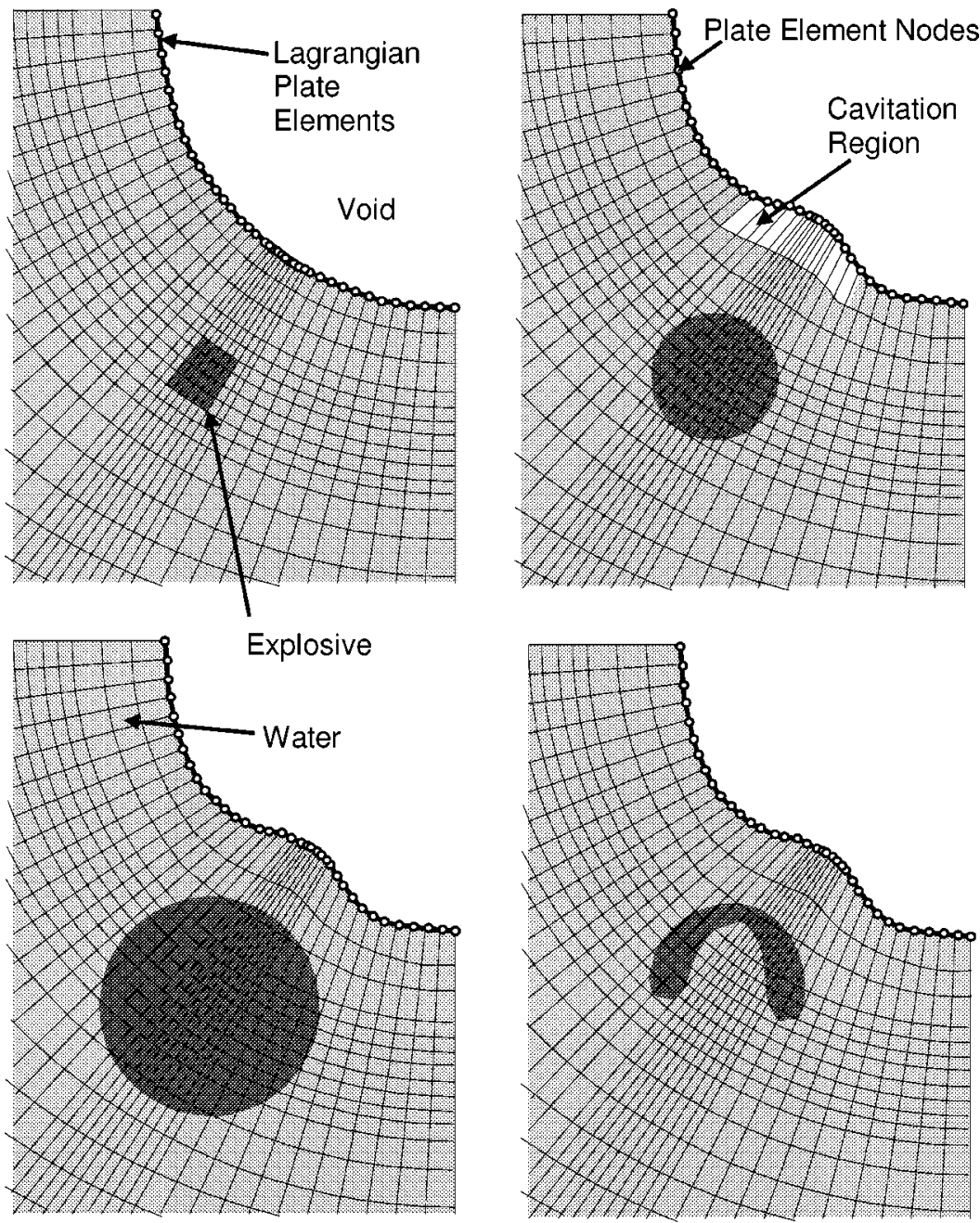

Fig. 10. Multimaterial ALE hydrocode UNDEX-SMI example. 


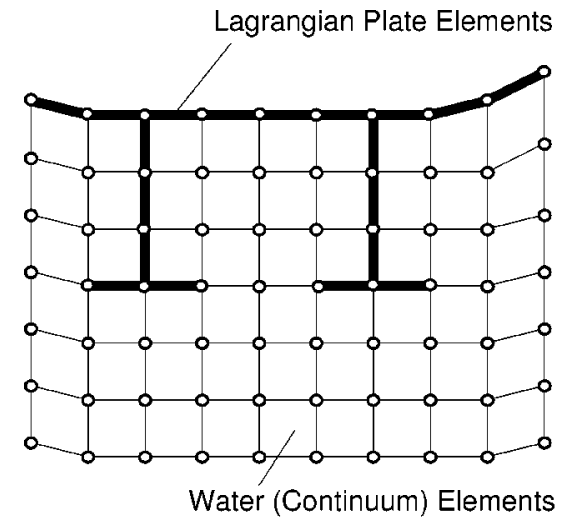

Initial Configuration

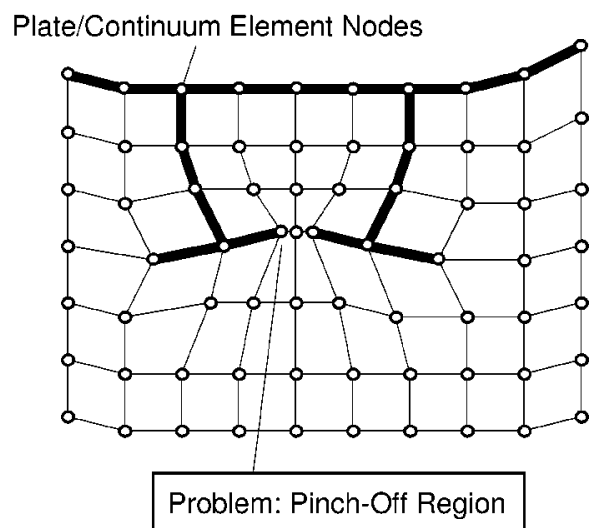

Later Configuration

Fig. 11. A difficult coupling problem for ALE codes.

Table 1

Descriptions and potential SMI applicabilities of prominent 3D hydrocodes

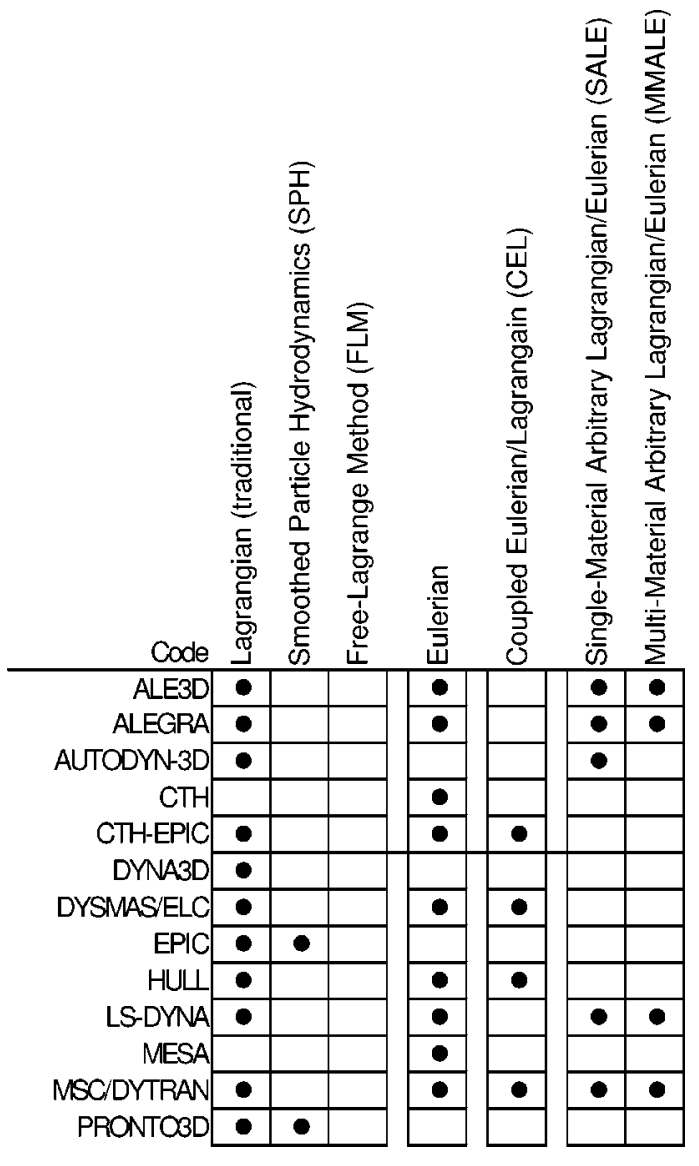

ure are some of these classes; underwater explosion structure-medium interaction is a combination.

Several hydrocode methods are in general use: Lagrangian, Eulerian, Coupled Eulerian-Lagrangian (CEL) and Arbitrary Lagrangian-Eulerian (ALE) and their derivatives. All hydrocodes are theoretically capable of solving problems in structure-medium interaction (SMI). However, the lack of infinite computer resources and the availability of Lagrangian "structural elements" allow the assessment of methods to be based on practicality rather than capability. Only those codes that include structural elements (i.e., thin plates, etc.) as well as fluid dynamics modeling capability, including detonation physics, are capable of practical SMI analysis of thin-walled structures. Prominent three-dimensional hydrocodes are so categorized in Table 1 .

General capabilities of the various hydrocode methods are summarized in Table 2. Since hydrocodes (like most other analytical tools) provide no measure of accuracy, analysis capability is defined as the possibility of generating a model that will produce useful results.

Traditional Lagrangian hydrocodes with structural elements, though applicable to structure-medium interaction, are not suited to model the large material distortions prevalent in longer-duration, bubblestructure interactions, and are therefore of limited utility. Nontraditional Lagrangian hydrocode methodologies (Free-Lagrangian, Smoothed Particle Hydrodynamics, and Total Lagrangian) show promise for UNDEX-SMI, though they are not as mature as the standard method.

Eulerian hydrocodes, though practical for modeling large distortion continuum dynamics, can be dismissed 
Table 2

Potential SMI applicabilities of hydrocode methods

\begin{tabular}{|c|c|c|c|c|}
\hline Method & 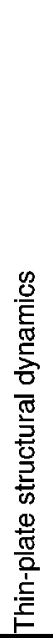 & 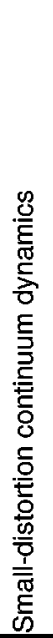 & 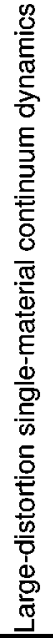 & 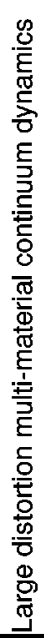 \\
\hline \multicolumn{5}{|l|}{ Lagrangian hydrocodes with structural elements } \\
\hline \multirow{4}{*}{$\begin{array}{r}\text { Traditional Lagrangian } \\
\text { Free-Lagrange Method (FLM) } \\
\text { Smoothed Particle Hydrodynamics (SPH) } \\
\text { Total Lagrangian formulation }\end{array}$} & $\bullet$ & 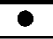 & & \\
\hline & $\bullet$ & $\bullet$ & - & $\bullet$ \\
\hline & $\bullet$ & $\bullet$ & $\bullet$ & $\bullet$ \\
\hline & $\bullet$ & 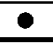 & - & \\
\hline Eulerian hydrocodes & & $\bullet$ & $\bullet$ & $\bullet$ \\
\hline $\begin{array}{l}\text { Coupled Eulerian/Lagrangain (CEL) hydrocodes with } \\
\text { structural elements }\end{array}$ & $\bullet$ & $\bullet$ & $\bullet$ & $\bullet$ \\
\hline \multicolumn{5}{|l|}{$\begin{array}{l}\text { Arbitrary Lagrangian/Eulerian (ALE) hydrocodes with } \\
\text { structural elements }\end{array}$} \\
\hline Single-Material ALE (SALE) & - & $\bullet$ & $\bullet$ & \\
\hline Multi-Material ALE (MMALE) & $\bullet$ & $\bullet$ & $\bullet$ & $\bullet$ \\
\hline
\end{tabular}

as impractical for UNDEX-SMI analyses, due to their inability to practically model thin-walled structures.

The Coupled Eulerian-Lagrangian (CEL) hydrocodes were developed for structure-medium interaction computations, specifically fluid-structure interaction, in which both the structure and its surrounding medium are modeled within their traditional frames of reference: Eulerian for fluid dynamics and Lagrangian for structural dynamics. CEL hydrocodes with structural elements are therefore practical for UNDEX-SMI analyses; such models have been demonstrated.

The Arbitrary Lagrangian-Eulerian (ALE) hydrocodes, developed with many of the same goals as the CEL codes, overcome some difficulties inherent in the CEL codes associated with coupling the Eulerian and Lagrangian coordinate systems, but introduce others. Multimaterial ALE (MMALE, as distinguished from Single-material ALE (SALE or SMALE)) hydrocodes with structural elements are potentially applicable to UNDEX-SMI analyses, though few models have been demonstrated with this relatively new method.
In summary, the simulation of underwater explosion structure-medium interaction with hydrocodes is practical. Of the four hydrocode methodologies (Lagrangian, Eulerian, Coupled Eulerian-Lagrangian, and Arbitrary Lagrangian-Eulerian), only the Eulerian method is not practical for UNDEX-SMI analysis. All other methods are applicable to various extents, and then only if the codes under consideration employ structural elements (i.e., thin plates and beams). The most versatile methods are the CEL and Multi-Material ALE.

\section{Acknowledgements}

This update of previous work was sponsored by the Office of the Secretary of Defense, Deputy Director, Operational Test and Evaluation, Live Fire Testing (Mr. James F. O'Bryon). The Office of Naval Research sponsored the original work while the author was employed by the Naval Surface Warfare Center, Indian 
Head Division (White Oak). The author is indebted to numerous reviewers; in particular Stephen Zilliacus of the Naval Surface Warfare Center, Carderock Division, and Vera Revelli of Sandia National Laboratories, California.

\section{References}

[1] F.L. Addessio, D.E. Carroll, J.K. Dukowicz, F.H. Harlow, J.N. Johnson, B.A. Kashiwa, M.E. Maltrud and H.M. Ruppel, CAVEAT: A computer code for fluid dynamics problems with large distortion and internal slip, Los Alamos National Laboratory LA-10613-MS-REVISED, 1990.

[2] R.T. Allen, M.G. Lumsden and J.E. Zerkle, Water shock waves in shallow water, Defense Nuclear Agency technical report DNA-TR-93-112, 1994

[3] U. Andelfinger, Simulations of underwater explosions against submerged structures using the DYSMAS/ELC code - Part A, in: Proc. of the 65th Shock and Vibration Symposium, Vol. II, 1994, p. 243.

[4] C.E. Anderson, Jr., An overview of the theory of hydrocodes, Int. J. of Impact Engineering 5 (1987), 33-59.

[5] S. Anderson, E. Dube, I. Otero and R. Sharp, Users manual for ALE3D - an arbitrary Lagrange/Eulerian 3D code system, Lawrence Livermore National Laboratory, 1993.

[6] S.W. Attaway, Update of PRONTO 2D and PRONTO 3D transient solid dynamics program, Sandia National Laboratories report SAND90-0102, 1990.

[7] R.L. Bell et al., CTH users manual and input instructions, Version 1.025, Sandia National Laboratories report SAND88-0523 (Revised), 1992.

[8] T. Belytschko, S.-H. Lee, I-S. Yeh, J.I. Lin, C.-S. Tsay and J.M. Kennedy, Adaptivity in crashworthiness calculations, Shock and Vibration 1(2) (1993/1994), 97-106.

[9] D.J. Benson, Computational methods in Lagrangian and Eulerian hydrocodes, Computer Methods in Applied Mechanics and Engineering 99 (1992), 235-394.

[10] W. Benz, Smooth particle hydrodynamics: A review, HarvardSmithsonian Center for Astrophysics, Preprint Series No. 2884, 1989.

[11] V.L. Bergmann, Transient dynamic analysis of plates and shells with PRONTO 3D, Sandia National Laboratories report SAND91-1182, 1991.

[12] J.U. Brackbill and J.J. Monaghan, eds, Particle methods in fluid dynamics and plasma physics, in: Proc. Workshop on Particle Methods in Fluid Dynamics and Plasma Physics, Los Alamos, NM, April 13-15, 1987; Comput. Phys. Comm. 48(1) (1988).

[13] Century Dynamics, AUTODYN Users's Manual Version 2.1, Century Dynamics, Oakland, CA, 1989.

[14] J.E. Chisum and Y.S.Shin, Multimaterial Eulerian and coupled Lagrangian-Eulerian finite element analysis of underwater shock problems, Naval Postgraduate School report NPSME-95-001, 1995.

[15] Y.W. Choi, Structure-occupant integrated analysis of an automobile equipped with an airbag, Cray Channels 16(2) (1994), 212-226.
[16] P.D. Church, I.G. Cullis, P.R. Greenwood and M.A. Nash, The Eulerian continuum mechanics code GRIM, Part 1: Technical Guide; Part 2: User Guide, Defence Research Agency reports DRA/WS/ET2/CR9351/1.0 and DRA/WS/ET2/CR9416/1.0, 1994 (Limited distribution).

[17] R.A. Clegg, J. Sheridan, C.J. Hayhurst and N.J. Francis, The application of SPH Techniques in AUTODYN-2D to kinetic energy penetration impacts on multi-layered soil and concrete targets, in: 8th Int. Symposium on Interaction of the Effects of Munitions with Structures, April 22-25 1997, Virginia, USA.

[18] R. Couch and D. Faux, Simulation of underwater explosion benchmark experiments with ALE3D, Lawrence Livermore National Laboratory report UCRL-CR-123819, 1996.

[19] R. Couch, R. McCallen, I. Otero and R. Sharp, 3D metal forming applications of ALE techniques, in: Proc. of the 5th Int. Conf. on Numerical Methods in Industrial Forming Processes - NUMIFORM '95, Shen and Dawson, eds, Balkema, Rotterdam, 1995, pp. 401-407.

[20] R. Couch et al., ALE3D Collaboration Site, http://www.llnl. gov/bdiv/ale3d/, 1997.

[21] D. Crawford, CTH reference manual: Explicit heat conduction, Sandia National Laboratories report SAND95-2623, 1995.

[22] P. Du Bois, Past, present, and future of industrial crashworthiness analysis, Cray Channels 12(1) (1990), 2-5.

[23] J.K. Dukowicz, M.C. Cline and F.L. Addessio, A general topology Godunov method, J. of Computational Physics 82 (1989), 29-63.

[24] B. Engelmann and J.O. Hallquist, NIKE2D - A nonlinear, implicit, two-dimensional finite element code for solid mechanics - user manual, Lawrence Livermore National Laboratory report UCRL-MA-105413, 1991.

[25] ESI, PAM-CRASH user's manual, theory manual, example manual - Version 10.2, Eng. System, 1989.

[26] A.V. Farnsworth Jr., CTH reference manual: Cell thermodynamics modifications and enhancements, Sandia National Laboratories report SAND95-2394, 1995.

[27] B. Fiessler, D.K. Han and A.B. Wardlaw, Jr., Coupled hydrocode simulation of the Seneca lake flat plate test, in: Proc. of the 66th Shock and Vibration Symposium, 1995, pp. 219-227.

[28] T. Frank and K. Gruber, Numerical simulation of frontal impact and frontal offset collisions, Cray Channels 13(4) (1992), 2-6.

[29] The Free-Lagrange Method, Lecture Notes in Physics, Vol. 236, M.J. Fritts, W.P Crowley and H. Trease, eds, Springer Verlag, Berlin, Germany, 1985.

[30] P.C. Galbraith, M.J. Finn and S.R. MacEwen, Evaluation of an LS-DYNA3D model for deep drawing of aluminum sheet, Cray Channels 13(4) (1992), 10-14.

[31] P.C. Galbraith and M. Finn, Supercomputer simulation of automotive sheet-metal forming, Cray Channels 16(2) (1994), 16-21.

[32] M.L. Gittings, TRIX: A free-Lagrangian hydrocode, SAIC internal report.

[33] P. Groenenboom, ed., MSC/PISCES User's Manual, Version 30, The MacNeal-Schwendler, Los Angeles, CA, 1991.

[34] J.O. Hallquist, User's manual for DYNA2D - An explicit twodimensional hydrodynamic finite element code with interactive rezoning and graphical display, Lawrence Livermore National Laboratory report UCID-18756, Rev. 3, 1988. 
[35] J.O. Hallquist, LS-DYNA3D theoretical manual, Livermore Software Technology Corporation report 1018, 1991.

[36] J.O. Hallquist and D.W. Stillman, VEC/DYNA3D user's manual, Livermore Software Technology Corporation report 1018, 1991.

[37] S. Hancock, PISCES 2DELK theoretical manual, Physics International, 1985.

[38] D.L. Hicks and R.T. Walsh, Numerical and computational analysis of the partial differential equations in hydrocodes and wavecodes, Sandia National Laboratories report SAND-750448, 1976

[39] HKS, ABAQUS user's manual, Vols I \& II, Version 4.8, Theory manual, Verification manual, Example problems manual, Hibbitt, Karlsson \& Sorensen, Pawtucket, RI, 1992.

[40] HKS, ABAQUS explicit user's manual, Version 5.1, Hibbitt, Karlsson \& Sorensen, Pawtucket, RI, 1992.

[41] K.S. Holian et al., MESA: A 3-D computer code for armor/antiarmor applications, in: Proc. of the Supercomputing World Conf., San Francisco, 1989.

[42] K.S. Holian et al., TECOLOTE: An object-oriented framework for hydrodynamics physics, in: Proc. for the Int. Workshop on New Models and Numerical Codes for Shock Wave Processes in Condensed Media; see also http://www-xdiv.lanl.gov/XCI/ PROJECTS/BLANCA/OPEN/oxford.html, 1997.

[43] H. Huang and K.C. Kiddy, Transient interaction of a spherical shell with an underwater explosion shock wave and the subsequent pulsating bubble, in: Dynamic Response of Structures to High-Energy Excitations, American Society of Mechanical Engineers AMD-Vol. 127/PVP-Vol. 225, 1991, pp. 1-11.

[44] IABG, The DYSMAS program family, B-TF-V197/3, IABG, Munich, Germany, 1992.

[45] G.R. Johnson, E.H. Petersen and R.A. Stryk, Incorporation of an SPH option into the EPIC code for a range of high velocity impact computations, in: High Velocity Impact Symposium, 1992.

[46] G.R. Johnson, Linking of Lagrangian particle methods to standard finite element methods for high velocity impact computations, in: Post-SMIRT Impact IV Seminar, Berlin, Germany, 1993.

[47] G.R. Johnson, R.A. Stryk, E.H. Petersen, T.J. Holmquist, J.A. Schonhardt and C.R. Burns, User instructions for the 1995 version of the EPIC code, Alliant Techsystems, 1994.

[48] W.E. Johnson and C.E. Anderson, History and application of hydrocodes in hypervelocity impact, Int. J. of Impact Engineering 5 (1987), 423-439.

[49] J.N. Johnson, C.L. Mader and M.S. Shaw, 2DL: A Lagrangian two-dimensional finite-difference code for reactive media, Los Alamos Scientific Laboratory report LA-8922-M, August 1981.

[50] K.-K. Kan and J.H. Stuhmiller, The phenomena of underwater explosion bubbles under a circular plate, JAYCOR final report under contract No. N60921-92-C-0048, 1994.

[51] R. Keene and A. Prior, MSC/DYTRAN user's manual, Part DT/A/000/UM, July 1991, update 7/2/92, The MacNealSchwendler.

[52] G.I. Kerley, CTH reference manual: The equation of state package, Sandia National Laboratories report SAND91-0344, 1991.
[53] G.I. Kerley, CTH equation of state package: Porosity and reactive burn models, Sandia National Laboratories report SAND92-0553, 1992.

[54] S.W. Key, HONDO - A finite element computer program for the large deformation response of axisymmetric solids, Sandia National Laboratory report 74-0039, 1974.

[55] D.B. Kothe, J.R. Baumgardner, S.T. Bennion, J.H. Cerutti, B.J. Daly, K.S. Holian, E.M. Kober, S.J. Mosso, J.W. Painter, R.D. Smith, W.H. Spangenberg and M.D. Torrey, A parallel finitedifference Eulerian method for transient three-dimensional multi-material deformation and fluid flow, Los Alamos National Laboratory Report LA-UR-93-2400, 1993.

[56] Y.W. Kwon, P.K. Fox and Y.S. Shin, Response of a cylindrical shell subject to a near field side-on explosion, in: Proc. 62nd Shock and Vibration Symposium, 1991, pp. 483-492.

[57] LANL, http://www-xdiv.lanl.gov/XTA/rageman/features/history.html, 1996.

[58] H. Lenselink and E. de Vries, Analysis of the loading of an aluminum cylinder by an underwater explosion, in: MSC World Users' Conf., June 20-24, 1994, Lake Buena Vista, Florida.

[59] L.D. Libersky, A.G. Petschek, T.C. Carney, J.R. Hipp and F.A. Allahdadi, High strain Lagrangian hydrodynamics - A threedimensional SPH code for dynamic material response, $J$. of Computational Physics 109 (1993), 67-75.

[60] R.W. Logan, F.J. Tokarz and R.G. Whirley, Crashworthiness: Planes, trains, and automobiles, Energy \& Technology Review (1993), 19-34.

[61] LSTC, LS-DYNA user's manual, Version 940, Livermore Software Technology Corporation, 1997.

[62] G. Luttwak, K. Florie and A. Venis, Numerical simulation of soft body impact, in: Shock Compression of Condensed Matter - 1991, North-Holland, Amsterdam, 1992, p. 999.

[63] H.U. Mair, H. Huang, B.J. Andrys, Jr. and M.E. Giltrud, Lagrangian hydrocode modeling of underwater explosive/target interaction, in: Proc. 61st Shock and Vibration Symposium, Vol. V, NAVSWC MP91-120, 1990, pp. 79-89.

[64] B.N. Maker, R.M. Ferencz and J.O. Hallquist, NIKE3D - A nonlinear, implicit, three-dimensional finite element code for solid and structural mechanics - user's manual, Lawrence Livermore National Laboratory report UCRL-MA-105268, 1991.

[65] D.A. Mandell, T.F. Adams, S.J. Mosso, K.S. Holian, F.L. Addessio and J.R. Baumgardner, MESA: A 3-D computer code for armor/anti-armor applications, Los Alamos National Laboratory report LA-UR-89-1263, 1989.

[66] D.A. Matuska and J. Osborn, HULL documentation: Volume II, User's manual, Orlando Technologies, Technical Report, 1986.

[67] J.M. McGlaun, CTH reference manual: Lagrangian step for hydrodynamic materials, Sandia National Laboratories report SAND90-2645, 1990.

[68] J.M. McGlaun, CTH reference manual: Cell thermodynamics, Sandia National Laboratories report SAND91-0002, 1991.

[69] J.M. McGlaun, S.L. Thompson and M.G. Elrick, CTH: A threedimensional shock wave physics code, Int. J. of Impact Engineering 10 (1990), 351-360.

[70] J.M. McGlaun, S.L. Thompson, L.N. Kmetyk and M.G. Elrick, A brief description of the three-dimensional shock wave physics code CTH, Sandia National Laboratories report SAND89-0607, 1990. 
[71] J.M. McGlaun and P. Yarrington, Large deformation wave Codes, in: High Pressure Shock Compression of Solids, J.R. Asay and M. Shahinpoor, eds, Springer-Verlag, 1993.

[72] R.M. McKeown, R. Grande and J.M. Mentges, Simulations of underwater explosions against submerged structures using the DYSMAS/ELC code - Part B: Analysis of an unstiffened cylinder, in: Proc. of the 65th Shock and Vibration Symposium, Vol. II, 1994, p. 253.

[73] MSC, MSC/NASTRAN user's manual - Version 66, The MacNeal-Schwendler, Los Angeles, CA, 1990.

[74] J.R. Neely, R. Couch, S. Futral and E. Dube, A parallel 3D ALE code for metal forming analyses, in: Proc. of the 5th Int. Conf. on Numerical Methods in Industrial Forming Processes - NUMIFORM '95, Shen and Dawson, eds, Balkema, Rotterdam, 1995, pp. 475-481.

[75] W.F. Noh, CEL: A time-dependent, two-space-dimensional, coupled Eulerian-Lagrange code, in: Methods in Computational Physics, Fundamental Methods in Hydrodynamics, Vol. 3, Academic Press, New York, 1964, pp. 117-179.

[76] J.S. Peery, K.G. Budge, M.K. Wong and T.G. Trucano, RHALE: A 3-D MMALE code for unstructured grids, in: ASME Winter Annual Meeting, 1993.

[77] A.G. Petschek and L.D. Libersky, Cylindrical smoothed particle hydrodynamics, J. of Computational Physics 109 (1993), 76-83.

[78] W.E. Pfrang, H. Luetje and J. Freercks, Shock response of a mine hunter due to sea ground mine explosions - numerical simulations, in: Proc. of the 59th Shock and Vibration Symposium, Vol. II, 1988, pp. 337-351.

[79] W. Pohl, M.J.v.d. Hoek, J.P. Buis, C.J.L. Florie, S.L. Hancock, P.H.L. Groenenboom, D.J.E. Hesselink, H. Lenselink and P.J.J. Schaffers, The PISCES software for defense: A survey of PISCES code applications for defense-orientated computational problems, PISCES International, Revision 2, 1984.

[80] J.K. Prentice, Zapotec: A code to perform coupled Eulerian/Lagrangian solid dynamics hydrocode calculations, http://www.quetzalcoatl.com/zapotec.html.

[81] J.C.W. Rogers and W.G. Szymczak, Computations of violent surface motions: computations with theory and experiment, Philosophical Trans. of the Royal Society of London, Series A, 355 (1997), 649-663.

[82] I.S. Sandler, A new computational procedure for wave propagation problems and a new procedure for non-reflecting boundaries, Computer Methods in Applied Mechanics and Engineering 164(1-2) (1998), 223-233.

[83] H.J. Schittke, W. Mohr, H. Luetje, W.E. Pfrang, J. Freercks and E. Niessen, The program DYSMAS/ELC and its application on underwater shock loading of vessels, in: Proc. of the 60th Shock and Vibration Symposium, Vol. IV, 1989, pp. 55-78.

[84] S.A. Silling, CTH reference manual: Viscoplastic models, Sandia National Laboratories report SAND91-0292, 1991.

[85] S.A. Silling, CTH reference manual: Johnson-Holmquist ceramic model, Sandia National Laboratories report SAND920576, 1992.

[86] S.A. Silling, CTH reference manual: Boundary layer algorithm for sliding interfaces in two dimensions, Sandia National Laboratories report SAND93-2487, 1994.
[87] R.M. Summers, J.S. Peery, M.K. Wong, E.S. Hertel, T.G. Trucano and L.C. Chhabildas, Recent progress in ALEGRA development and application to ballistic impacts, Int. J. of Impact Engineering 20 (1997), 779-788.

[88] J.W. Swegle, TOODY IV - A computer program for twodimensional wave propagation, Sandia National Laboratories report SAND-78-0552, September 1978.

[89] J.W. Swegle, ARTOO - An automatically rezoning twodimensional Lagrangian finite difference wavecode, Sandia National Laboratories report SAND81-2235, 1981.

[90] J.W. Swegle and S.W. Attaway, On the feasibility of using smoothed particle hydrodynamics for underwater explosion calculations, Sandia National Laboratories report SAND950311, 1995 .

[91] W.G. Szymczak, Computations and experiments of pressure loadings from underwater explosion bubbles, Structures under Extreme Loading Conditions, ASME PVP-Vol. 351, 1997, pp. 17-27.

[92] L.M. Taylor and D.P. Flanagan, PRONTO 2D - A twodimensional transient solid dynamics program, Sandia National Laboratories report SAND86-0594, 1987.

[93] L.M. Taylor and D.P. Flanagan, PRONTO 3D - A threedimensional transient solid dynamics program, Sandia National Laboratories report SAND87-1912, 1989.

[94] P.A. Taylor, CTH reference manual: The Steinberg-GuinanLund viscoplastic model, Sandia National Laboratories report SAND92-0716, 1992.

[95] P.A. Taylor, CTH reference manual: The transverse isotropic (TI) model, Sandia National Laboratories report SAND952750, 1995.

[96] P.A. Taylor, CTH reference manual: The Bammann-ChiesaJohnson viscoplastic/damage model, Sandia National Laboratories report SAND96-1626, 1996.

[97] R. Tipton, CALE user's manual, Lawrence Livermore National Laboratory, 1992.

[98] A.B. Wardlaw, Jr., B. Fiessler and D.K. Han, Hydrocode simulation of the seneca lake flat plate test, in: Proc. of the 65th Shock and Vibration Symposium, Vol. III, 1994, pp. 81-89.

[99] R.G. Whirley and J.O. Hallquist, DYNA3D - A nonlinear, explicit, three-dimensional finite element code for solid and structural mechanics - user manual, Lawrence Livermore National Laboratory report UCRL-MA-107254, 1991.

[100] M. Wilkins, Calculation of elastic-plastic flow, in: Methods in Computational Physics, Fundamental Methods in Hydrodynamics, Vol. 3, Academic Press, 1964, pp. 211-263.

[101] P. Yarrington, The CTH-EPIC link - A coupled Eulerian/Lagrangian computational method, in: Proc. of the 12th U.S. National Congress on Computational Mechanics, 1994.

[102] P. Yarrington and J.K. Prentice, A coupled Eulerian/Lagrangian method for computational continuum dynamics analysis, in: Program of the 1995 APS Topical Conf. Shock Compression of Condensed Matter, Bulletin of the American Physical Society 40(6) (1995), 1388. 

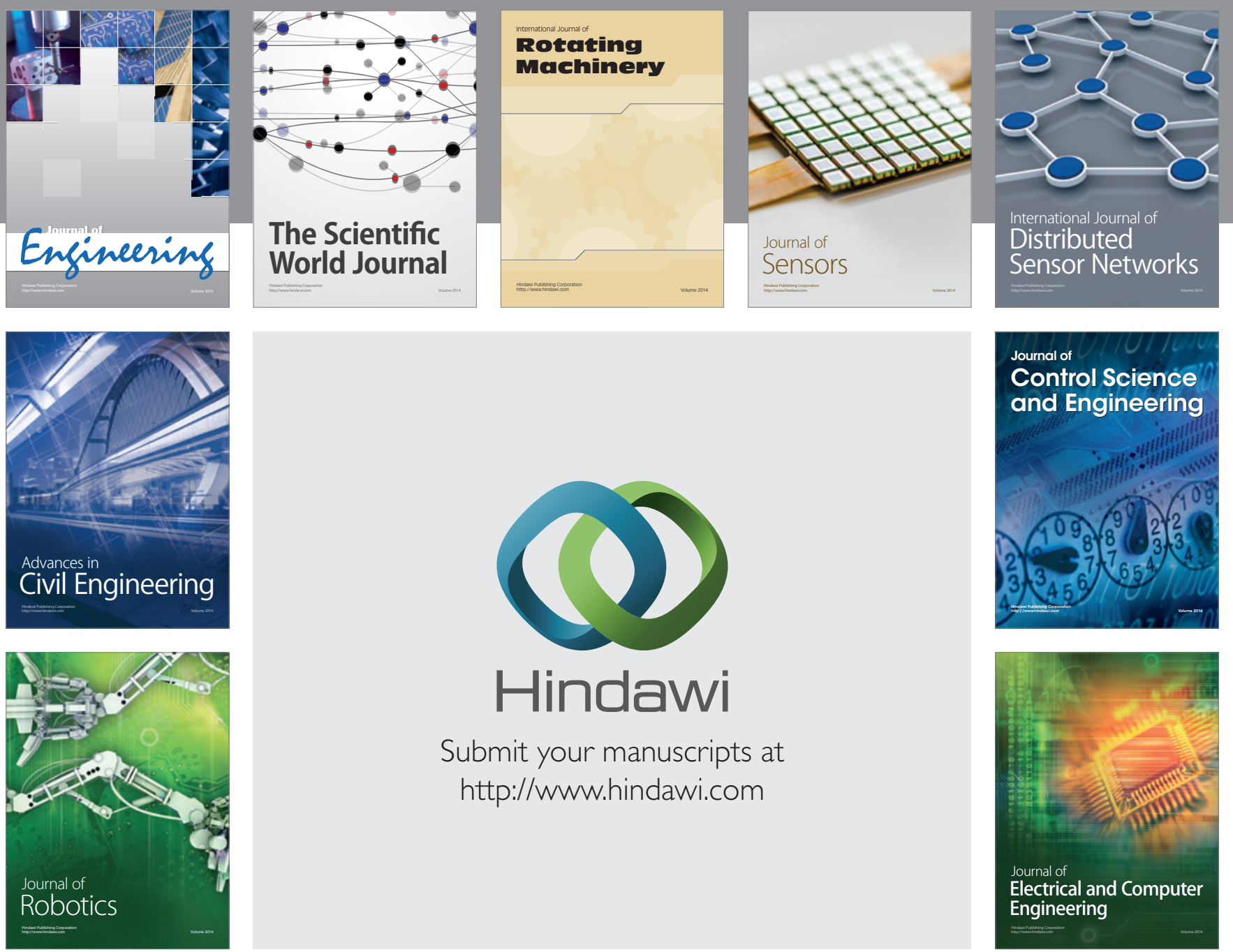

Submit your manuscripts at

http://www.hindawi.com
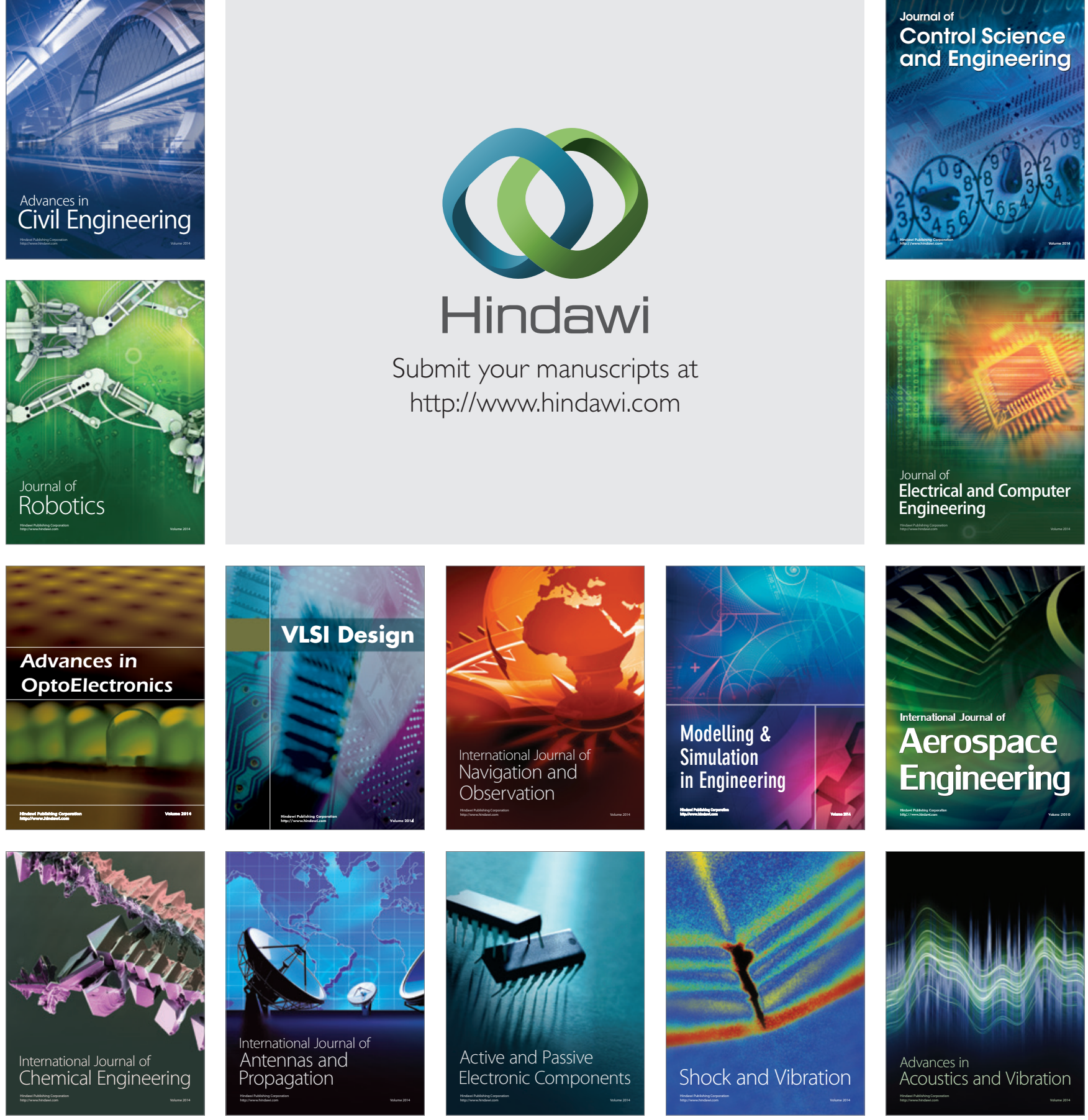